\title{
Exome sequencing-based molecular autopsy of formalin-fixed paraffin-embedded tissue after sudden death
}

\author{
Richard D. Bagnall, PhD ${ }^{1,2}$, Jodie Ingles, MPH, $\mathrm{PhD}^{1,2,3}$, Laura Yeates, BSc ${ }^{1,3}$, Samuel F. Berkovic, MD \\ and Christopher Semsarian, MBBS, PhD ${ }^{1,2,3}$
}

\begin{abstract}
Purpose: Sudden death in the young is a devastating complication of inherited heart disorders. Finding the precise cause of death is important, but it is often unresolved after postmortem investigation. The addition of postmortem genetic testing, i.e., the molecular autopsy, can identify additional causes of death. We evaluated DNA extracted from formalin-fixed paraffin-embedded postmortem tissue for exome sequencing-based molecular autopsy after sudden death in the young.
\end{abstract}

Methods: We collected clinical and postmortem information from patients with sudden death. Exome sequencing was performed on DNA extracted from fixed postmortem tissue. Variants relevant to the cause of death were sought.

Results: Five patients with genetically unresolved sudden death were recruited. DNA extracted from fixed postmortem tissue was degraded. Exome sequencing achieved 20-fold coverage of at least $82 \%$ of coding regions. A threefold excess of singleton variants was found in the exome sequencing data of one patient. We found a de novo SCN1A frameshift variant in a patient with sudden unexpected death in epilepsy and a LMNA nonsense variant in a patient with dilated cardiomyopathy.

Conclusion: DNA extracted from fixed postmortem tissue is applicable to exome sequencing-based molecular autopsy. Fixed postmortem tissues are an untapped resource for exome-based studies of rare causes of sudden death.

Genet Med advance online publication 23 March 2017

Key Words: exome sequencing; fixed postmortem tissue; molecular autopsy; sudden death

\section{INTRODUCTION}

Sudden cardiac death in the young is a devastating outcome of a number of inherited cardiomyopathies and primary arrhythmia syndromes. ${ }^{1}$ Population-based estimates in studies of sudden cardiac death in persons aged 1 to 35 years show an annual incidence of 1.3 to 2.8 per 100,000 persons..$^{2-4}$ It is important to establish the precise cause of sudden cardiac death in the young because most inherited heart diseases show autosomal dominant inheritance, placing first-degree relatives at $50 \%$ risk of harboring a disease-causing variant. Postmortem investigation can identify a cause of death in the majority of sudden cardiac death in the young; however, the death remains unexplained in $40 \%$ of cases. ${ }^{2}$ Fatal inherited primary arrhythmia syndromes, including the long QT syndrome, are not evident in postmortem investigation because they are associated with a structurally normal heart. Noncardiac genetic conditions may also cause unexplained sudden death; for example, sudden unexpected death in epilepsy (SUDEP) is the leading cause of epilepsy-related mortality. ${ }^{5}$

The addition of postmortem genetic testing, i.e., the molecular autopsy, identifies causes of death beyond those detected in postmortem investigation alone. ${ }^{2,6}$ Finding a pathogenic variant in a patient with unexplained sudden death can clarify the cause of death and enable cascade genetic testing of asymptomatic first-degree family members. Consensus guidelines recommend that a molecular autopsy be considered in cases of unexplained sudden cardiac death in the young. ${ }^{7}$ Owing to the practical limitations of screening genes one exon at a time, the molecular autopsy initially included the major genes of the long QT syndrome (KCNH2, KCNQ1, and SCN5A) and a subset of the 105 exons of the RYR2 gene. ${ }^{6,8-10}$ Massively parallel sequencing technologies can simultaneously screen large panels of genes, including all 22,000 protein-coding genesi.e., the exome-and have expanded the scope of the molecular autopsy. ${ }^{11,12}$ Screening all epilepsy and cardiac arrhythmia genes with exome sequencing-based molecular autopsy has also provided novel insights into genetic risk factors of SUDEP. ${ }^{11}$

Postmortem blood or extracted DNA is not always available for a molecular autopsy, especially in historical cases, but formalin-fixed and paraffin-embedded tissue (fixed tissue) is usually prepared for histological examination and more widely available. This approach prevents further tissue autolysis and preserves tissue morphology; however, it leads to DNA-protein crosslinking and DNA degradation, both of which inhibit polymerase chain reaction (PCR) amplification. ${ }^{13}$ For these reasons, DNA extracted from fixed postmortem tissue has typically been

${ }^{1}$ Agnes Ginges Centre for Molecular Cardiology, Centenary Institute, Sydney, Australia; ${ }^{2}$ Sydney Medical School, University of Sydney, Sydney, Australia; ${ }^{3}$ Department of Cardiology, Royal Prince Alfred Hospital, Sydney, Australia; ${ }^{4}$ Epilepsy Research Centre, Department of Medicine, University of Melbourne, Austin Health, Melbourne, Australia. Correspondence: Christopher Semsarian (c.semsarian@centenary.org.au) 
used to selectively test cosegregation of a specific variant previously found in another affected family member or to genetically screen a limited number of gene regions in short overlapping PCR amplicons. The ability to perform comprehensive genetic analysis using fixed postmortem tissue has the potential to resolve many previously unexplained cases of sudden death in the young. Emerging studies indicate that fixed tumor tissue is a promising source of DNA for exome sequencing: $;{ }^{14-16}$ however, in general, autolysis of postmortem tissue is more advanced before fixation than for surgical tumor specimens. We sought to evaluate the feasibility of exome sequencing-based molecular autopsy of patients with sudden death using DNA isolated from fixed postmortem tissue.

\section{MATERIALS AND METHODS}

\section{Patients}

We recruited five families referred to the Genetic Heart Diseases Clinic at Royal Prince Alfred Hospital, Sydney, following the sudden death of a family member for whom fixed postmortem tissue blocks were the only available source of DNA. A cardiologist reviewed the deceased's available medical records, family history, and postmortem investigation report and performed clinical evaluation of consenting first-degree family members. Next of kin, or parents in the case of children, gave informed consent to request fixed postmortem tissue and perform exome sequencing-based genetic testing of the decedent. All studies were carried out with the strict approval of and in accordance with the Sydney Local Health District Ethics Review Committee, Australia.

\section{Methods \\ DNA isolation. Fixed spleen or liver tissue blocks from the deceased were requested from the coroner. The fixed-tissue blocks were between 3 and 14 years old; the time from death to tissue fixation, the fixation and embedding protocol, and storage conditions were unknown. The block with the largest surface area of tissue was selected for each patient, and 2010 - $\mu \mathrm{m}$ sections were cut using a microtome (Leica Biosystems, Nussloch, Germany) and transferred to a microcentrifuge tube. Paraffin wax was dissolved with three 10 -min washes with $1 \mathrm{ml}$ xylene and the residual cell pellet was digested for $48 \mathrm{~h}$ in 200 $\mu \mathrm{l}$ cell lysis buffer (Qiagen, Hilden, Germany) with $10 \mathrm{mg} / \mathrm{ml}$ proteinase $\mathrm{K}$ at $56^{\circ} \mathrm{C}$. DNA was extracted using a QIAmp DNA mini kit (Qiagen) according to the manufacturer's instructions and resuspended in $100 \mu \mathrm{l}$ elution buffer. Five microliters of DNA was resolved in $1 \%$ agarose gel for $40 \mathrm{~min}$. DNA was quantified using Picogreen (Invitrogen, Carlsbad, CA) fluorescence- based quantification.}

Exome sequencing and variant calling. A DNA library was prepared using the SureSelectXT Library Prep Kit (Agilent, Santa Clara, CA), enriched for the SureSelectXT Human All Exon v5 exome (Agilent), and paired-end 101-bp sequenced using an Illumina HiSeq 4000 platform (Macrogen, Seoul, Republic of Korea). The 101-bp sequences were aligned to the human genome reference sequence (GRCh37/hg19/b37) using BWA v0.7.12 $2^{17}$ with the default parameters. Sequence alignment files were converted to binary using SamTools v0.1.19 ${ }^{18}$ and sorted with concurrent duplicate read removal using Novosort v1.03.01 (Novocraft, Selangor, Malaysia). The Genome Analysis Tool Kit v3.3.0 (GATK) was used for read realignment around short insertions and deletions (indels), base quality score recalibration, and genotyping of single-nucleotide variants (SNVs) and indels, using haplotype caller in gVCF mode, according to the best practices (http://www.broadinstitute.org/gatk/guide/best-practices). Variants were filtered for quality using variant quality score recalibration tranche level, calculated using the known SNV sites from HapMap v3.3, dbSNP and the Omni chip array from the 1000 Genomes Project. To "pass," SNVs and indels were required to achieve a tranche of $99.8 \%$ and $99 \%$, respectively. SNVs and indels were annotated using the SeattleSeq Annotation v8.07 server (http://snp.gs.washington.edu/ SeattleSeqAnnotation137/index.jsp) and compared against the November 2010 release of the 1000 Genomes Project data (http://www.1000genomes.org/), 6,500 exomes of the Exome Variant Server, the National Heart, Lung, and Blood Institute GO Exome Sequencing Project, Seattle, WA (http://evs. gs.washington.edu/EVS/), Exome Aggregation Consortium (ExAC) v0.3.1 data (ftp://ftp.broadinstitute.org/pub/ExAC_ release), and in-house exome sequences of 363 individuals with diverse cardiac pathologies.

Comparison exome group. To assess the quality of variant calls, we compared fixed-tissue exome data for the 5 patients who were of European ancestry with data for 112 unrelated patients of European ancestry who had cardiac disease and were recruited to our research program for various studies. The comparison exome group had DNA isolated from fresh peripheral blood lymphocytes, were exome-sequenced using the same enrichment kit and protocol, and were analyzed using the same analysis framework as for the fixed-tissue exome data. Singleton variants were counted for each individual using PLINK/Seq v0.10 (https://atgu.mgh.harvard.edu/plinkseq/) with the command "pseq exome i-stats" and the genomic positions of singletons were retrieved with the command "pseq exome v-view --geno --only-minor --mask mac=1-1."

Filtering and prioritization of variants. An overview of the variant filtration strategy is shown in Supplementary Figure S1 online. We retained SNVs causing a nonsynonymous, splice-site, or nonsense substitution and indels occurring in the coding regions or splice signal sequences with (i) a minor allele frequency of $<0.1 \%$ in the 1000 Genomes Project Data and in 6,500 exomes from the National Heart, Lung, and Blood Institute exome sequencing project; (ii) an allele count of $<50$ in the ExAC data; and (iii) an allele count of $<20$ in our inhouse exome data. Variants were sought in panels of genes relevant to the cause of death, i.e., 42 cardiomyopathy genes in patients with hypertrophic or dilated cardiomyopathy, 42 
cardiomyopathy genes plus 23 cardiac arrhythmia genes in patients with unexplained sudden death, and 72 epilepsy genes plus 23 cardiac arrhythmia genes in patients with SUDEP (Supplementary Table S1 online). The pathogenicity of remaining rare variants was assessed using the standards and guidelines of the American College of Medical Genetics and Genomics. ${ }^{19}$

Sanger validation of variants. PCR primers were designed using Primer3Plus (http://www.bioinformatics.nl/cgi-bin/ primer3plus/primer3plus.cgi/) to amplify regions up to $200 \mathrm{bp}$ surrounding variants of interest. Following standard PCR amplification, PCR amplicons were visualized on 2\% agarose gel; excess primers and deoxynucleotide triphosphates were removed using alkaline phosphatase (New England Biolabs, Ipswich, MA) and exonuclease I (New England Biolabs), respectively, and were Sanger DNA-sequenced (Macrogen). Sequencing electropherograms were manually inspected using Sequencher v5.1 (Gene Codes, Ann Arbor, MI).

\section{RESULTS}

\section{Clinical and postmortem findings}

Table 1 shows the clinical characteristics and key postmortem findings for five patients with sudden death for whom fixed postmortem tissue blocks were the only available source of DNA. Patient 1 was diagnosed with Dravet syndrome (severe myoclonic epilepsy of infancy) during life and she died at age 2 years in her sleep. There was a family history of hypertrophic cardiomyopathy and sudden cardiac death on the paternal side and a previously identified 4-bp duplication of MYBPC3 (rs397516019, NM_000256.3:c.3476_3477dupTTAT), causing a Phe1161Tyrfs ${ }^{*} 9$ frameshift and premature termination of translation segregated with disease in three clinically affected family members. This variant was also found in a clinically unaffected aunt who was 46 years of age. Patient 1 had not been genetically tested for the MYBPC3 variant. Postmortem neuropathological examination of the brain showed mild cerebral swelling, subcortical gliosis, and minor hippocampal asymmetry. Histology of heart sections showed a focus of mixed inflammatory cells in the left ventricle. The cause of death was SUDEP.

Patient 2 had a clinical diagnosis of severe dilated cardiomyopathy with an ejection fraction of $15 \%$, biatrial enlargement, and atrial fibrillation. His mother presented with atrial fibrillation at age 50 years and was subsequently diagnosed with mild dilated cardiomyopathy. Her DNA was not available for genetic testing at the beginning of the study. The sister of patient 2 was clinically normal at age 35 years. Patient 2 died suddenly in the shower at age 38 years, 6 months after his clinical diagnosis of dilated cardiomyopathy; a limited postmortem examination confirmed dilated cardiomyopathy.

Patient 3, who was asymptomatic, died suddenly during strenuous exercise at age 23 years. At postmortem investigation, his left ventricle was hypertrophic and the interventricular septum measured $27 \mathrm{~mm}$. The cause of death was hypertrophic cardiomyopathy. Both parents and three siblings, aged 35-40 years, were clinically screened and found to be clinically unaffected.

Patient 4 died suddenly at age 33 years. At postmortem investigation, his heart weighed $750 \mathrm{~g}$ and had significant left ventricular hypertrophy that measured $30 \mathrm{~mm}$. Histology of the left ventricle showed marked muscle fiber enlargement and disarray, with a small patchy area of fibrosis. In addition, there was mild to moderate triple vessel coronary artery disease with generalized atherosclerosis of the aorta; histology showed wellestablished atheroma in the left anterior descending coronary artery. Although the macroscopic and histological findings are diagnostic of hypertrophic cardiomyopathy, the possible contribution of coronary artery disease to the cause of death is difficult to determine. The autopsy report to the coroner recorded the cause of death as possible hypertrophic cardiomyopathy.

Patient 5 was an otherwise healthy male who died in his sleep at age 16 years. There was no family history of cardiac disease. The postmortem investigation, including histology and toxicology analysis, was normal and the cause of death was unexplained sudden death.

\section{Exome analysis of fixed postmortem tissue}

Fixed postmortem tissue from the five patients collected from 2002 to 2013 yielded 1.3 to $4.1 \mu \mathrm{g}$ DNA (concentration 13.3 to $40.7 \mathrm{ng} / \mu \mathrm{l})$ from $2010-\mu \mathrm{m}$ sections and showed smearing on agarose gel electrophoresis, indicating DNA degradation (Supplementary Figure S2 online). Exome sequencing yielded 56.9-73.6 million reads per patient, with $>82 \%$ of the consensus coding sequence version 15 (CCDSv15) covered by at least 20 reads (range 82.6-90.7\%; Supplementary Table S2 online). On average, the cardiomyopathy, cardiac arrhythmia, and epilepsy genes on which the current study focused had $88 \%$ of coding regions covered at least 20 times (Supplementary Table S1 online). To assess the quality of variant calls, we aggregated exome data for the 5 fixed-tissue patients who were of European ancestry with data for 112 unrelated patients of European ancestry who had cardiac disease. There were no significant differences in the total number of variants between individuals. When singleton variants were counted, i.e., a variant found only once among all 117 individuals, patient 4 had more singletons $(n=1,373)$ than any other individual (median $=361 ; \mathrm{SD}$ = 115.6; Supplementary Figure S3 online), and 450 (33\%) of his singletons were within $15 \mathrm{bp}$ of another singleton, i.e., clustered singletons. Visual inspection of the clusters of singletons showed specific reads containing multiple variants, suggestive of sequencing artifacts (Supplementary Figure S4 online).

Table 2 shows the rare variants found in genes relevant to the cause of death of each patient. Patient 1 died of SUDEP, and we found a single base duplication of SCN1A (NM_001165963.1:c.2274dup) causing a His759Thrfs ${ }^{\star} 13$ frameshift and premature termination of translation. The variant was Sanger-verified in patient 1 and was absent from both parents, indicating a de novo mutation (Figure 1). The SCN1A frameshift variant has not been previously reported. Only two 
Table 1 Patient characteristics and relevant postmortem investigation findings

\begin{tabular}{|c|c|c|c|c|c|c|c|c|}
\hline $\begin{array}{l}\text { Patient } \\
\text { no. }\end{array}$ & Sex & $\begin{array}{l}\text { Age at } \\
\text { death } \\
\text { (years) }\end{array}$ & $\begin{array}{l}\text { Year of } \\
\text { death }\end{array}$ & $\begin{array}{l}\text { Relevant } \\
\text { medical history }\end{array}$ & $\begin{array}{l}\text { Relevant family } \\
\text { history }\end{array}$ & $\begin{array}{l}\text { Circumstances } \\
\text { of death }\end{array}$ & $\begin{array}{l}\text { Relevant postmortem } \\
\text { investigation findings }\end{array}$ & Cause of death \\
\hline 1 & Female & 2 & 2010 & Dravet syndrome & $\begin{array}{l}\text { Hypertrophic } \\
\text { cardiomyopathy } \\
\text { and sudden } \\
\text { cardiac death }\end{array}$ & $\begin{array}{l}\text { Found deceased } \\
\text { in bed }\end{array}$ & $\begin{array}{l}\text { Cerebral swelling, subcortical } \\
\text { gliosis, hippocampal asymmetry; } \\
\text { foci of inflammatory cells in cardiac } \\
\text { interstitium }\end{array}$ & $\begin{array}{l}\text { Sudden } \\
\text { unexpected } \\
\text { death in epilepsy }\end{array}$ \\
\hline 2 & Male & 38 & 2013 & $\begin{array}{l}\text { Dilated } \\
\text { cardiomyopathy } \\
\text { and atrial } \\
\text { fibrillation }\end{array}$ & $\begin{array}{l}\text { Dilated } \\
\text { cardiomyopathy; } \\
\text { atrial fibrillation } \\
\text { in mother }\end{array}$ & $\begin{array}{l}\text { Found } \\
\text { unconscious in } \\
\text { shower }\end{array}$ & $\begin{array}{l}\text { Heart weight } 584 \mathrm{~g} \text {; left and right } \\
\text { ventricular diameter } 60 \mathrm{~mm} \text { and } \\
45 \mathrm{~mm} \text {, respectively; patchy myocyte } \\
\text { hypertrophy, interstitial fibrosis }\end{array}$ & $\begin{array}{l}\text { Dilated } \\
\text { cardiomyopathy }\end{array}$ \\
\hline 3 & Male & 23 & 2006 & None & $\begin{array}{l}\text { Five first-degree } \\
\text { relatives clinically } \\
\text { normal }\end{array}$ & $\begin{array}{l}\text { Collapsed } \\
\text { during physical } \\
\text { training }\end{array}$ & $\begin{array}{l}\text { Interventricular septum } 27 \mathrm{~mm} \text {; } \\
\text { myocyte disarray; atheroma of the } \\
\text { aortic valve ring. }\end{array}$ & $\begin{array}{l}\text { Hypertrophic } \\
\text { cardiomyopathy }\end{array}$ \\
\hline 4 & Male & 33 & 2002 & $\begin{array}{l}\text { History of mild } \\
\text { hypertension }\end{array}$ & Unknown & Not reported & $\begin{array}{l}\text { Maximal left ventricular wall thickness } \\
30 \mathrm{~mm} \text {; myocardial fibrosis; muscle } \\
\text { fiber enlargement and disarray; mild } \\
\text { to moderate triple vessel coronary } \\
\text { artery disease }\end{array}$ & $\begin{array}{l}\text { Possible } \\
\text { hypertrophic } \\
\text { cardiomyopathy }\end{array}$ \\
\hline
\end{tabular}

Table 2 Rare variants in genes implicated in the cause of sudden death

\begin{tabular}{|c|c|c|c|c|c|c|}
\hline Patient no. & Cause of death & Gene & cDNA variation & Consequence & $\begin{array}{c}\text { ExAC allele } \\
\text { count }\end{array}$ & CADD score \\
\hline \multirow[t]{3}{*}{2} & DCM & NEXN & NM_001172309.1:C.394C>T & Arg132Cys & 10 & 11 \\
\hline & & $\pi N$ & NM_001256850.1:c.35635+1G>A & Abolish splice donor & 4 & 23 \\
\hline & & LMNA & NM_170707.3:c.1401G>A & Trp467* & 0 & 36 \\
\hline
\end{tabular}

CADD, combined annotation-dependent depletion; DCM, dilated cardiomyopathy; ExAC, Exome Aggregation Consortium; HCM, hypertrophic cardiomyopathy; SUDEP sudden unexpected death in epilepsy.

SCN1A null variants are found in more than 60,000 population controls (probability loss-of-function-intolerant $=1.0$ ), and they are a nonsense variant in the last exon and a canonical splice site variant. In contrast, more than 330 mutations of SCN1A have been reported in Dravet syndrome and approximately two-thirds are frameshift and nonsense variants. ${ }^{20} \mathrm{We}$ classified the de novo SCNA1 His759Thrfs ${ }^{\star} 13$ frameshift as pathogenic (Supplementary Table S3 online). Patient 1 had a family history of hypertrophic cardiomyopathy and sudden death, and an MYBPC3 frameshift variant was found through previous genetic testing of the family. The exome data showed that patient 1 did not harbor the MYBPC 3 variant, and this was Sanger-confirmed (Figure 1).

In patient 2 , who had a clinical diagnosis of dilated cardiomyopathy with atrial fibrillation, we found a Trp467* nonsense variant in LMNA (NM_170707.3:c.1401G>A; Figure 2). This variant was previously reported in the Clinvar database (RCV000182396) as pathogenic in an unrelated patient with dilated cardiomyopathy. There is only one LMNA nonsense variant in more than 60,000 population controls, which leads to a premature termination of translation of 10 amino acids from the end of the protein. Loss-of-function LMNA variants account for up to $8 \%$ of dilated cardiomyopathy ${ }^{21,22}$ and a higher proportion of dilated cardiomyopathy with conduction disease. ${ }^{23}$ We classified the LMNA Trp $467^{\star}$ variant as pathogenic (Supplementary Table S3 online). We also found two rare missense variants in NEXN and a rare canonical splice site variant in TTN (Table 2). All four variants were Sanger-verified in patient 2 and only the LMNA variant was found in his clinically affected mother (Figure 2). NEXN is a candidate pathogenic gene for dilated cardiomyopathy with an excess of variants in patients with dilated cardiomyopathy compared to controls. ${ }^{24,25}$ The NEXN variants were assumed to be in cis because they were both absent in the mother. The Ile483Thr variant was predicted to be tolerated by PolyPhen 2 and SIFT, whereas the Arg132Cys variant was predicted to be damaging by PolyPhen2, SIFT, and Mutation Taster. The TTN splice site variant occurs in a highly expressed 81-bp exon in dilated cardiomyopathy (proportion spliced-in $=1.0)^{26}$ and encodes 27 amino acids from the PEVK repeat domain. We classified the NEXN and TTN variants as uncertain clinical significance (Supplementary Table S3 online). In patient 4 , who had a postmortem diagnosis of possible hypertrophic cardiomyopathy, we Sanger-verified a novel TMEM43 missense variant (NM_024334.2:c.766G>A) causing 


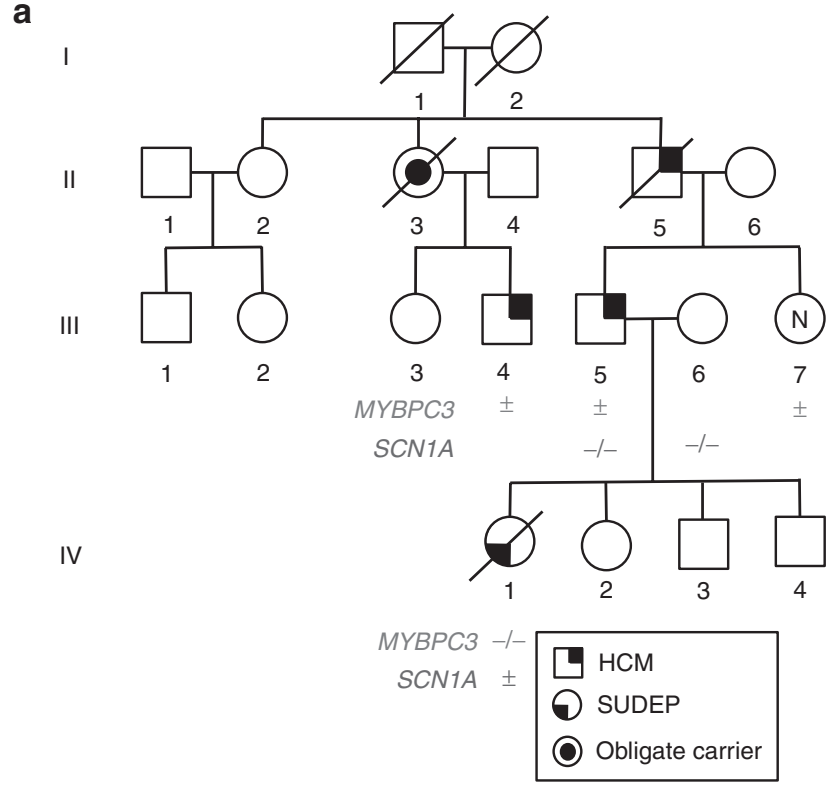

b

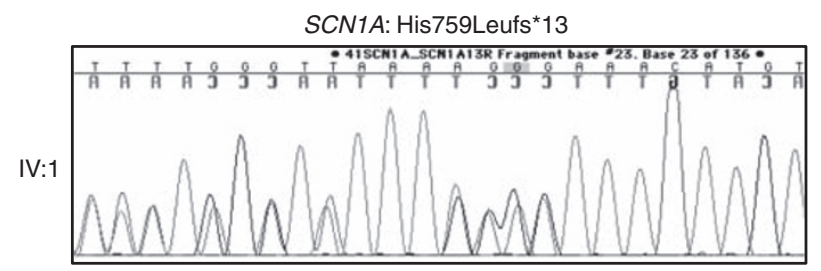

Figure 1 Family 1 pedigree and sequencing analysis of patient 1. (a) Family pedigree showing genotypes of MYBPC3 (blue) and SCN1A (red) variants. +/- heterozygous; -/- homozygous reference allele; $N$, clinically normal. (b) Sanger verification of SCN1A frameshift variant.

a Gly256Ser substitution of uncertain clinical significance. No rare variants were found in 42 cardiomyopathy genes in patient 3 , who had a postmortem diagnosis of hypertrophic cardiomyopathy, or in 65 cardiomyopathy and cardiac arrhythmia genes in patient 5, whose death was unexplained following postmortem investigation.

\section{DISCUSSION}

We have extracted DNA from fixed postmortem tissue stored for up to 14 years from five patients with sudden death and performed exome sequencing-based molecular autopsy. We identified a pathogenic variant in two patients that confirmed their clinical diagnoses and impacted the clinical management of surviving family members. Our study offers encouragement that archival fixed postmortem tissue is a source of DNA for exome sequencing-based molecular autopsy when postmortem blood is not available. Collections of archival fixed postmortem tissue are an untapped source of DNA for exome sequencingbased studies of rare causes of sudden death in patients who otherwise could not be sequenced.

Our study highlights the value of identifying a genetic cause of sudden death and the widespread impact of being able to use fixed postmortem tissue for the source of DNA. We found a pathogenic single base duplication of $S C N 1 A$ in patient 1 , who
I

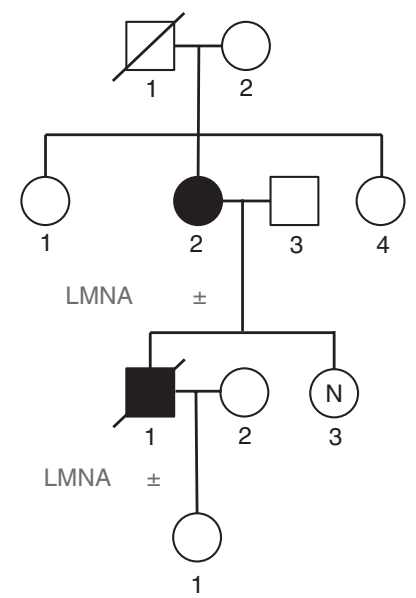

b

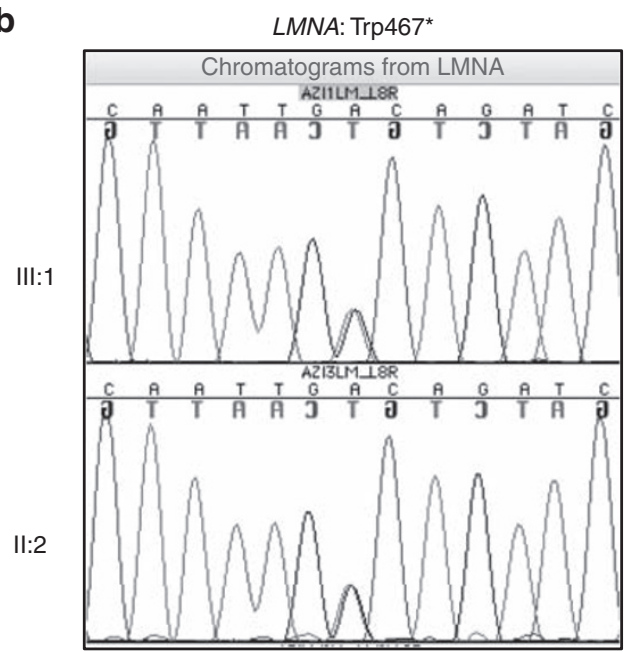

Figure 2 Family 2 pedigree and sequencing analysis of LMNA nonsense variant. (a) Family pedigree showing genotypes of LMNA variant. +/- heterozygous; N, clinically normal. (b) Sanger sequencing electropherograms of $L M N A$ variant.

had Dravet syndrome and died with sudden unexpected death in epilepsy. With Dravet syndrome, more than $80 \%$ of patients have a de novo SCN1A pathogenic variant and there is a high mortality rate, with half of deaths due to SUDEP. ${ }^{20}$ Genotyping parental DNA established that the novel duplication occurred de novo; therefore, genetic analysis of patient 1 was the only opportunity to find the pathogenic variant and that had relevance to clinical management of the family because there was a very low risk of her three siblings developing Dravet syndrome. Exome sequencing-based analysis of SCN1A was more efficient than Sanger sequencing, which would require PCR amplification of all 28 exons of SCN1A in small overlapping segments to overcome the challenges of amplifying degraded fixed-tissue DNA. The exome data were further used to establish that patient 1 did not inherit a MYBPC3 variant from her father and that her death was unrelated to the family history of hypertrophic cardiomyopathy and sudden cardiac death.

Genetic testing of dilated cardiomyopathy is challenging because more than 100 genes have been described, including 
the very large Titin gene. ${ }^{27}$ A pathogenic LMNA nonsense variant was found in patient 2 and his pacemaker-dependent mother; both had dilated cardiomyopathy and atrial fibrillation. Although the cause of dilated cardiomyopathy with atrial fibrillation is consistent with a dominant $L M N A$ nonsense variant, the NEXN and TTN variants found in patient 2 may have provided an additive genetic contribution to his more severe phenotype. DNA of the mother was not available at the start of the study and fixed postmortem tissue of patient 2 was the only available source of DNA for genetic testing. Finding the cause of DCM has relevance to ongoing clinical management of the family with presymptomatic cascade genetic testing, which is now an option for the daughter of patient 2 , who is currently age 2 years.

A pathogenic variant was not found in patients 3 and 4, who had a postmortem diagnosis of hypertrophic cardiomyopathy, or in patient 5 with unexplained sudden death. We did find a novel TMEM43 Gly256Ser missense variant in patient 4; however, pathogenic TMEM43 variants are a rare cause of arrhythmogenic right ventricular cardiomyopathy ${ }^{28,29}$ and the characteristic feature of fibro-fatty replacement of cardiomyocytes was absent.

Standardized guidelines for postmortem investigation of sudden death of the young have not been endorsed in many countries and a blood sample for a molecular autopsy is not always collected. We recommend that a postmortem blood sample for genetic testing should be kept for all sudden cardiac death of the young. In Australia and New Zealand, best practice guidelines regarding postmortem investigation of sudden death of a young person now mandate that tissue or blood suitable for DNA extraction must be obtained ${ }^{7,30}$; nevertheless, postmortem blood may not be available for a number of reasons. Patient 2 had a limited postmortem investigation performed at the request of the next of kin. Postmortem blood of patients 3 and 4 had not been collected at postmortem investigation performed more than 10 years ago or was since disposed of. No blood remained after postmortem investigation of a child 2 years of age. As is routine during postmortem investigation, formalin-fixed tissue specimens are embedded for histological analysis and do not require specific storage conditions such as freezing, thereby reducing the burden of long-term storage.

Our results demonstrate that DNA extracted from fixed postmortem tissue can be exome-enriched and sequenced using a commercial sequencing provider, generate raw sequencing data that can be passed through a standard BWA/GATK analysis framework, cover CCDSv15 regions to levels comparable with that of DNA isolated from fresh peripheral blood lymphocytes, and yield SNV and indel genotypes that are clinically useful in the setting of a molecular autopsy. Tissue fixation is not without consequences to the integrity of DNA and may partly explain the excess of false-positive singletons in patient 4. In addition, the unknown storage conditions and extent of autolysis of the postmortem tissue before fixation may affect DNA quality. Genotypes of patients showing an excess of singletons should be excluded from exome-wide gene collapsing or variant burden statistical analyses that compare the number of rare variants between cases and controls to avoid erroneous gene associations.

There are common observations emerging from studies assessing the feasibility of exome sequencing with DNA extracted from fixed surgical tumor tissue. The quantity and quality of DNA extracted from fixed tumor tissue are low and are associated with increased specimen storage time. ${ }^{14}$ Van Allen et al. were able to exome sequence and extract DNA down to 16 ng from all 99 fixed tumor tissue samples, whereas Carrick et al. failed to extract sufficient DNA from 3 out of 59 fixed tumor tissue samples that had been stored for up to 32 years. ${ }^{14,16}$ Additional sequencing is required for DNA extracted from fixed tumor tissue to achieve coverage of target regions matching that of high-quality DNA because of a higher level of duplicate sequence reads and trimming of low-quality sequences. ${ }^{15,23}$ Furthermore, excessively fragmented DNA may be too short for bridge amplification on the Illumina sequencing platforms. Of the 135 cardiomyopathy, cardiac arrhythmia, and epilepsy genes on which we focused our analysis, on average, $88 \%$ of coding regions were covered with at least 20 sequence reads; however, some genes had low coverage. In particular, the key hypertrophic cardiomyopathy genes, MYH7, TNNI3, and MYBPC3 had 69,72 , and $83 \%$ coverage with at least 20 sequence reads. Further investigation of the genetically unsolved cause of hypertrophic cardiomyopathy for patients 3 and 4 should focus on the regions of low coverage in these genes. DNA extracted from fixed tissue would be suitable for sequencing of a targeted gene panel, which typically yields a higher sequencing read depth and coverage of target genes than exome sequencing. Gene panel sequencing may be considered when the phenotype is highly suggestive of one or more key causal genes; however, if no variant is found, then further sequencing is required to screen additional genes. The current study and previous studies have shown that sequence data from some fixed-tissue specimens have an excess of variants that are artifacts, likely introduced by formalin fixation. Comparing sequencing data of matched fixed tissue and fresh-frozen tissue from the same patient showed that genotypes with the highest confidence were called from regions with high sequence depth. ${ }^{14,15}$ Despite the challenges with using fixed tumor tissue stored for varying lengths of time and storage conditions, it is a potential source of DNA for massively parallel sequencing approaches when high-quality DNA is not available.

The molecular autopsy is a useful addition to postmortem investigation of sudden death in the young. The selection of genes to be tested should be guided by the phenotype when possible and additional information gained through clinical and genetic evaluations of family members may be vital for establishing the pathogenicity of variants. DNA extracted from fixed postmortem tissue offers the opportunity for a molecular autopsy for patients without an alternative source of DNA such as whole blood. Collections of fixed postmortem tissues are an unexploited source of DNA for exome analysis-based studies of patients with rare causes of sudden death that are otherwise difficult to recruit. Although postmortem whole blood is the ideal source for DNA extraction and genetic analysis, fixed 
postmortem tissues can facilitate meaningful exome-based genetic analysis when blood is not available. Resolving previously unexplained sudden death cases has many clinical benefits by providing families with a cause of death and opportunities to identify at-risk family relatives through cascade genetic testing, with the ultimate goal of preventing sudden death.

\section{SUPPLEMENTARY MATERIAL}

Supplementary material is linked to the online version of the paper at http://www.nature.com/gim

\section{ACKNOWLEDGMENTS}

J.I. is the recipient of a National Heart Foundation of Australia Future Leader Fellowship (100833). C.S. is the recipient of a National Health and Medical Research Council (NHMRC) Practitioner Fellowship (1059156). This study is funded in part by an NHMRC Project Grant (1046441).

\section{DISCLOSURE}

S.F.B. has received honoraria and/or payments for development of educational presentations from UCB Pharma, Novartis Pharmaceuticals, Sanofi-Aventis, and Janssen-Cilag. He has a patent for SCN1A testing held by Bionomics Inc and licensed to various diagnostic companies (no financial return).

\section{REFERENCES}

1. Semsarian C, Ingles J, Wilde AA. Sudden cardiac death in the young: the molecular autopsy and a practical approach to surviving relatives. Eur Heart $J$ 2015;36:1290-1296.

2. Bagnall RD, Weintraub RG, Ingles J, et al. A prospective study of sudden cardiac death among children and young adults. N Engl J Med 2016;374:2441-2452.

3. Papadakis M, Sharma S, Cox S, Sheppard MN, Panoulas VF, Behr ER. The magnitude of sudden cardiac death in the young: a death certificate-based review in England and Wales. Europace 2009;11:1353-1358.

4. Winkel BG, Holst AG, Theilade J, et al. Nationwide study of sudden cardiac death in persons aged 1-35 years. Eur Heart J 2011;32:983-990.

5. Neligan A, Bell GS, Johnson AL, Goodridge DM, Shorvon SD, Sander JW. The long-term risk of premature mortality in people with epilepsy. Brain 2011;134(Pt 2):388-395.

6. Skinner JR, Crawford J, Smith W, et al.; Cardiac Inherited Disease Group New Zealand. Prospective, population-based long QT molecular autopsy study of postmortem negative sudden death in 1 to 40 year olds. Heart Rhythm 2011;8:412-419.

7. Ackerman MJ, Priori SG, Willems S, et al.; Heart Rhythm Society (HRS); European Heart Rhythm Association (EHRA). HRS/EHRA expert consensus statement on the state of genetic testing for the channelopathies and cardiomyopathies: this document was developed as a partnership between the Heart Rhythm Society (HRS) and the European Heart Rhythm Association (EHRA). Europace 2011;13:1077-1109.

8. Chugh SS, Senashova O, Watts A, et al. Postmortem molecular screening in unexplained sudden death. J Am Coll Cardiol 2004;43:1625-1629.

9. Tester DJ, Ackerman MJ. Postmortem long QT syndrome genetic testing for sudden unexplained death in the young. J Am Coll Cardiol 2007;49:240-246.

10. Tester DJ, Spoon DB, Valdivia HH, Makielski JC, Ackerman MJ. Targeted mutational analysis of the RyR2-encoded cardiac ryanodine receptor in sudden unexplained death: a molecular autopsy of 49 medical examiner/coroner's cases. Mayo Clin Proc 2004;79:1380-1384.
11. Bagnall RD, Crompton DE, Petrovski S, et al. Exome-based analysis of cardiac arrhythmia, respiratory control, and epilepsy genes in sudden unexpected death in epilepsy. Ann Neurol 2016;79:522-534.

12. Loporcaro CG, Tester DJ, Maleszewski JJ, Kruisselbrink T, Ackerman MJ. Confirmation of cause and manner of death via a comprehensive cardiac autopsy including whole exome next-generation sequencing. Arch Pathol Lab Med 2014;138:1083-1089.

13. Srinivasan $M$, Sedmak $D$, Jewell $S$. Effect of fixatives and tissue processing on the content and integrity of nucleic acids. Am J Patho/ 2002;161:1961-1971.

14. Carrick DM, Mehaffey MG, Sachs MC, et al. Robustness of next generation sequencing on older formalin-fixed paraffin-embedded tissue. PLoS One 2015;10:e0127353.

15. Oh E, Choi YL, Kwon MJ, et al. Comparison of accuracy of whole-exome sequencing with formalin-fixed paraffin-embedded and fresh frozen tissue samples. PLoS One 2015;10:e0144162.

16. Van Allen EM, Wagle N, Stojanov P, et al. Whole-exome sequencing and clinical interpretation of formalin-fixed, paraffin-embedded tumor samples to guide precision cancer medicine. Nat Med 2014;20:682-688.

17. Li H, Durbin R. Fast and accurate long-read alignment with Burrows-Wheeler transform. Bioinformatics 2010;26:589-595..

18. Li H, Handsaker B, Wysoker A, et al.; 1000 Genome Project Data Processing Subgroup. The Sequence Alignment/Map format and SAMtools. Bioinformatics 2009;25:2078-2079.

19. Richards S, Aziz N, Bale S, et al.; ACMG Laboratory Quality Assurance Committee. Standards and guidelines for the interpretation of sequence variants: a joint consensus recommendation of the American College of Medical Genetics and Genomics and the Association for Molecular Pathology. Genet Med 2015;17:405-424.

20. Dravet C, Bureau M, Oguni H, Cokar O, Guerrini R. Dravet syndrome (Severe myoclonic epilepsy in infancy). In: Bureau M, Genton P, Dravet C, et al. (eds). Epileptic Syndromes in Infancy, Childhood and Adolescence, 5th edn. John Libbey Eurotext: Montrouge, France, 2012:125-56.

21. Parks SB, Kushner JD, Nauman D, et al. Lamin A/C mutation analysis in a cohort of 324 unrelated patients with idiopathic or familial dilated cardiomyopathy. Am Heart J 2008;156:161-169.

22. Taylor MR, Fain PR, Sinagra G, et al.; Familial Dilated Cardiomyopathy Registry Research Group. Natural history of dilated cardiomyopathy due to lamin AVC gene mutations. J Am Coll Cardio/ 2003;41:771-780.

23. van Tintelen JP, Hofstra RM, Katerberg H, et al.; Working Group on Inherited Cardiac Disorders, line 27/50, Interuniversity Cardiology Institute of The Netherlands. High yield of LMNA mutations in patients with dilated cardiomyopathy and/or conduction disease referred to cardiogenetics outpatient clinics. Am Heart J 2007;154:1130-1139.

24. Hassel D, Dahme T, Erdmann J, et al. Nexilin mutations destabilize cardiac Z-disks and lead to dilated cardiomyopathy. Nat Med 2009;15: 1281-1288.

25. Walsh R, Thomson KL, Ware JS, et al. Reassessment of Mendelian gene pathogenicity using 7,855 cardiomyopathy cases and 60,706 reference samples. Genet Med 2017;19:192-203.

26. Roberts AM, Ware JS, Herman DS, et al. Integrated allelic, transcriptional, and phenomic dissection of the cardiac effects of titin truncations in health and disease. Sci Trans/ Med 2015;7:270ra6.

27. Favalli V, Serio A, Grasso M, Arbustini E. Genetic causes of dilated cardiomyopathy. Heart 2016;15: 2015-308190.

28. Baskin B, Skinner JR, Sanatani S, et al. TMEM 43 mutations associated with arrhythmogenic right ventricular cardiomyopathy in non-Newfoundland populations. Hum Genet 2013;132:1245-1252.

29. Merner ND, Hodgkinson KA, Haywood AF, et al. Arrhythmogenic right ventricular cardiomyopathy type 5 is a fully penetrant, lethal arrhythmic disorder caused by a missense mutation in the TMEM43 gene. Am J Hum Genet 2008:82:809-821.

30. Skinner JR, Duflou JA, Semsarian C. Reducing sudden death in young people in Australia and New Zealand: the TRAGADY initiative. Med J Aust 2008;189: 539-540. 